\title{
Получение атомарно-чистых и структурно-упорядоченных поверхностей эпитаксиальных пленок CdTe для последующей эпитаксии
}

\author{
(C) А.С. Тарасов ${ }^{1}$, Н.Н. Михайлов ${ }^{1,2}$, С.А. Дворецкий ${ }^{1,3}$, P.В. Менщиков ${ }^{1}$, И.Н. Ужаков ${ }^{1}$, \\ A.C. Кожухов ${ }^{1}$, Е.В. Федосенко ${ }^{1}$, О.Е. Терещенко ${ }^{1,2}$ \\ ${ }^{1}$ Институт фризики полупроводников им. А.В. Ржанова \\ Сибирского отделения Российской академии наук, \\ 630090 Новосибирск, Россия \\ ${ }^{2}$ Новосибирский государственный университет, \\ 630090 Новосибирск, Россия \\ ${ }^{3}$ Томский государственный университет, \\ 634050 Томск, Россия \\ E-mail: tarasov1916@yandex.ru
}

Поступила в Редакцию 12 апреля 2021 г. В окончательной редакции 19 апреля 2021 г. Принята к публикации 19 апреля 2021 г.

Получена атомарно-чистая и структурно-упорядоченная поверхность слоя СdTе подложки (013) $\mathrm{GaAs} / \mathrm{ZnTe} / \mathrm{CdTe}$ после хранения на воздухе с помощью обработки в изопропиловом спирте, насыщенном парами соляной кислоты, и дальнейшего термического отжига в сверхвысоком вакууме. Показано, что химическая обработка поверхности СdTе приводит к удалению собственных оксидов и обогащению поверхности слоем элементного теллура. Во время прогрева в вакууме наблюдается две стадии изменения состояния поверхности $\left(\sim 125^{\circ} \mathrm{C}\right.$ и $\left.\leq 250^{\circ} \mathrm{C}\right)$. При $T>250^{\circ} \mathrm{C}$ происходят десорбция элементного теллура и формирование Те-стабилизированной структуры $(1 \times 1)$ CdTe $(013)$.

Ключевые слова: поверхность, подложка GaAs, $\mathrm{HgCdTe}, \mathrm{PbSnTe}$, РФЭС, ДБЭО, одноволновая эллипсометрия, химическая подготовка.

DOI: $10.21883 /$ FTP.2021.09.51289.18

\section{1. Введение}

Твердые растворы соединений II-VI $\left(\mathrm{Hg}_{1-x} \mathrm{Cd}_{x} \mathrm{Te}\right)$ и $\mathrm{IV}-\mathrm{VI}\left(\mathrm{Pb}_{1-x} \mathrm{Sn}_{x} \mathrm{Te}\right)$ являются узкозонными полупроводниками и широко применяются в качестве фоточувствительных материалов для приемников инфракрасного (ИК) и терагерцового (ТГц) излучения [1]. В последние годы интерес к этим соединениям еще больше вырос, что связано с открытием у них свойств топологических изоляторов (ТИ), в которых возникают электронные поверхностные состояния дираковского типа из-за сильного спин-орбитального взаимодействия, проявляющиеся в соединениях, содержащих тяжелые атомы [2]. Для выделения поверхностного транспорта в соединениях на основе $\mathrm{Hg}_{1-x} \mathrm{Cd}_{x} \mathrm{Te}$ и $\mathrm{Pb}_{1-x} \mathrm{Sn}_{x}$ Tе выращиваются квантовые структуры (КС), в которых проявляются новые физические эффекты, такие как квантовый эффект Холла, эффект поля и спин-вентильный эффект в ТИ и др. [2-8].

Для получения эпитаксиальных пленок твердых растворов $\mathrm{Hg}_{1-x} \mathrm{Cd}_{x} \mathrm{Te}, \mathrm{Pb}_{1-x} \mathrm{Sn}_{x}$ Те и КС широко используется метод молекулярно-лучевой эпитаксии (МЛЭ). Для достижения высокого кристаллического качества эпитаксиальных слоев необходимо обеспечить согласование по параметру кристаллической решетки границы пленка-подложка. Подложки из монокристаллического $\mathrm{Cd}_{1-x} \mathrm{Zn}_{x}$ Te c $x=0.04$ обеспечивают идеальное со- гласование параметров кристаллической решетки для выращивания слоев $\mathrm{Hg}_{1-x} \mathrm{Cd}_{x}$ Te с составом $x=0.22$. Однако высокая стоимость, трудности в получении подложек большой площади и неоднородность состава ограничивают использование материала в научных исследованиях и массовом производстве. В качестве альтернативы подложкам CdZnTe используются подложки из $\mathrm{GaAs}$ и $\mathrm{Si}$ с буферными слоями $\mathrm{ZnTe}$ и $\mathrm{CdTe}(\mathrm{GaAs}(\mathrm{Si}) / \mathrm{ZnTe} / \mathrm{CdTe})[9]$. Такие подложки хорошо согласованы с $\mathrm{HgCdTe}$ и обеспечивают высокое кристаллическое качество получаемых ИК приемников и КС.

$\mathrm{B}$ качестве подложки для выращивания слоев $\mathrm{PbTe}$ и $\mathrm{PbSnTe}$ используются объемные монокристаллы $\mathrm{BaF}_{2}$, которые получают сколом в вакууме, или эпитаксиальные пленки $\mathrm{BaF}_{2}$, выращенные на кремниевых подложках [10-15]. Однако рост пленок PbSnTe на $\mathrm{BaF}_{2}$ всегда начинается по островковому механизму, что делает невозможным создание тонких пленок без дополнительных буферных слоев [16].

Хорошее согласование параметров решеток CdTe и $\mathrm{PbSnTe}$ позволяет использовать альтернативные подложки $\mathrm{GaAs}(\mathrm{Si}) / \mathrm{ZnTe} / \mathrm{CdTe}$ для роста $\mathrm{PbSnTe}$. Однако использование таких подложек требует либо отдельной камеры для их роста, либо транспортировки через атмосферу. В последнем случае необходимо разработать метод подготовки поверхности CdTe перед эпитаксиальным 
ростом. В настоящее время для очистки поверхностей соединений III-V и IV-VI от собственных оксидов и углеродных загрязнений успешно используется обработка в безводном растворе соляной кислоты в изопропиловом спирте (HCl-iPA) [17-21]. При этом происходит пассивация поверхности слоем атомов элементов V или VI групп, который защищает поверхность от влияния атмосферы в течение 3-5 мин для загрузки подложки в установку МЛЭ и десорбируется при относительно низких температурах. Мы предполагаем, что обработка альтернативной подложки $\mathrm{GaAs}(\mathrm{Si}) / \mathrm{ZnTe} / \mathrm{CdTe}$ в раствоpe HCl-iPA может обеспечить удаление собственных оксидов и пассивацию поверхности теллуром, а последующий прогрев в сверхвысоком вакууме позволит получить атомарно-чистую и структурно-упорядоченную поверхность.

Таким образом, целью данной работы является изучение процессов подготовки атомарно-чистой и структурно-упорядоченной поверхности альтернативной подложки $\mathrm{GaAs} / \mathrm{ZnTe} / \mathrm{CdTe}$ при обработке в HCl-iPA и прогреве в сверхвысоком вакууме с последующим МЛЭростом слоев $\mathrm{HgCdTe}$ и $\mathrm{PbSnTe}$.

\section{2. Образцы и методика эксперимента}

Альтернативные подложки (013) GaAs/ZnTe/CdTe диаметром 2 дюйма были выращены методом МЛЭ на установке „Обь-М“ [22]. На подложке (013) GaAs последовательно выращивались буферные слои ZnTe и CdTe толщиной 0.03 мкм и 5.5-6.3 мкм соответственно.

Химическая обработка образцов проходила в несколько этапов: обработка в HCl-iPA в течение 10 c, промывка образца в чистом изопропиловом спирте, сушка образца в потоке сухого азота. Способ приготовления раствора $\mathrm{HCl}$-іРА описан в работе [17]. После химической обработки подложка в течение 3 мин загружалась в установку МЛЭ для выращивания соответствующих слоев или в камеру аналитической системы Nanoscan-50 (Cameca) для анализа состава поверхности.

Состояние поверхности контролировалось методами одноволновой эллипсометрии in situ [23] и дифракции быстрых электронов на отражение (ДБЭО) с энергией электронов 28 кэВ.

Для контроля элементного состава и состояния поверхности использовался метод рентгеновской фотоэлектронной спектроскопии (РФЭС) с линией рентгеновского излучения $\mathrm{Al} K_{\alpha}$ с энергией 1486.6 эВ и разрешением $\sim 1$ эВ.

\section{3. Результаты и обсуждение}

На рис. 1 изображены типичные спектры РФЭС линий $\mathrm{Te} 3 d_{5 / 2}$ и $\mathrm{Cd} 3 d_{3 / 2}$ для поверхности CdTe до и после химической обработки в растворе $\mathrm{HCl}-\mathrm{iPA}$, а также после обработки в HCl-iPA и термического отжига.

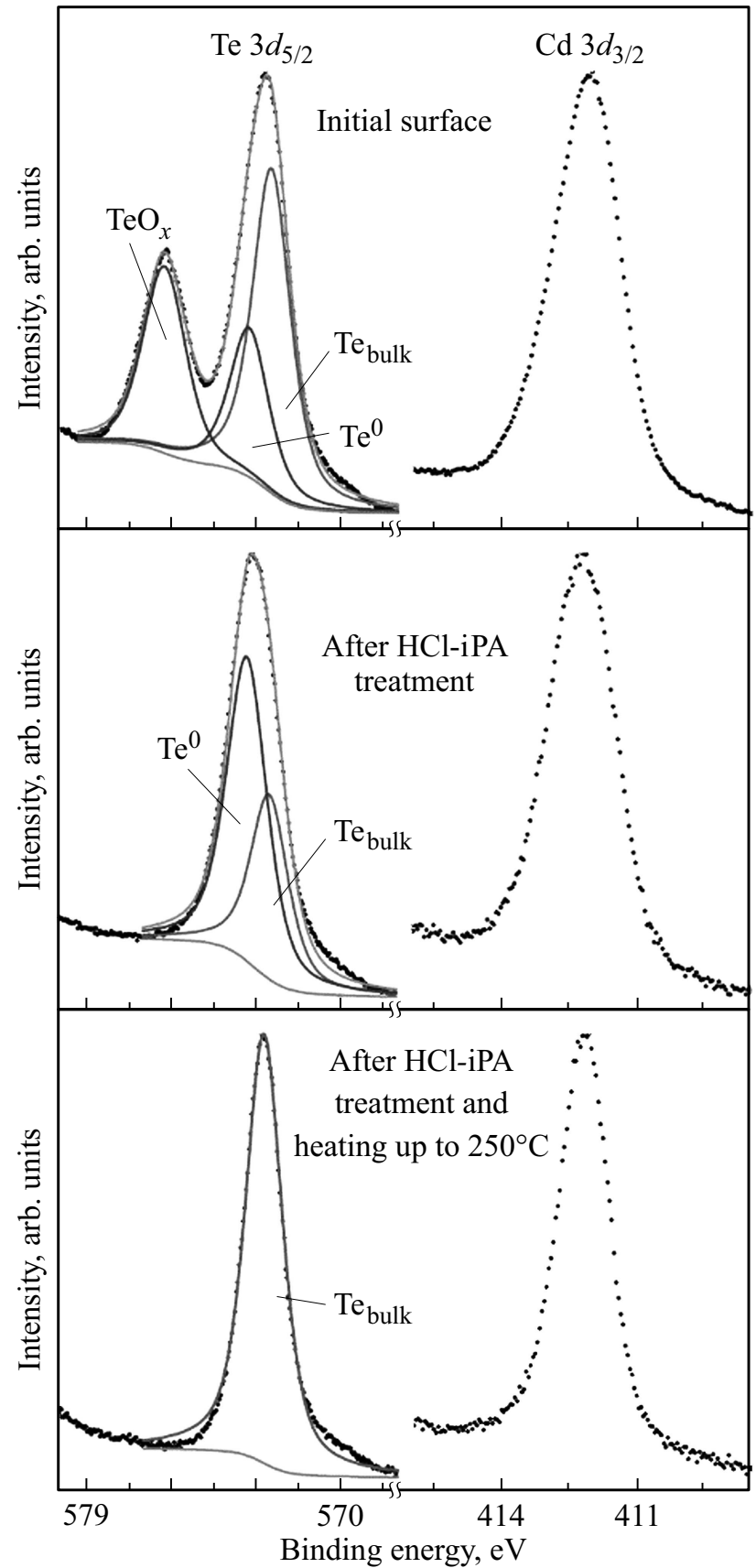

Рис. 1. Фотоэлектронные спектры поверхности CdTе гетероструктуры (013) GaAs/ZnTe/CdTe: исходная поверхность; после обработки в $\mathrm{HCl}-\mathrm{iPA}$; после обработки в $\mathrm{HCl}-\mathrm{iPA}$ и прогрева до $250^{\circ} \mathrm{C}$.

Положение компонент разложения линий спектров, значение ширины на полувысоте (ШПВ) и поверхностное соотношение компонентов $[\mathrm{Cd}] /[\mathrm{Te}+\mathrm{Cd}]$ приведены в таблице. Для исходной поверхности, после хранения подложки (013) GaAs/ZnTe/CdTe в атмосферных условиях, наблюдалось два пика линии Те $3 d_{5 / 2}$, один из которых имел асимметричную форму. Разложение такого спектра на три линии показало, что линия с энергией 576.3 эВ соответствует оксиду теллура $\mathrm{TeO}_{x}$. Линии с 
Энергии связи компонент пиков, ширины на полувысоте и соотношение интенсивностей для линий Те $3 d_{5 / 2}$ и Cd $3 d_{3 / 2}$

\begin{tabular}{|c|c|c|c|c|c|c|c|}
\hline \multirow{3}{*}{ Поверхность } & \multicolumn{4}{|c|}{ Энергия связи, эВ } & \multicolumn{2}{|c|}{ ШПВ, эВ } & \multirow{3}{*}[\mathrm{Cd}]{$/[\mathrm{Cd}+\mathrm{Te}]$} \\
\hline & \multicolumn{3}{|c|}{ Te $3 d_{5 / 2}$} & \multirow{2}{*}{$\mathrm{Cd} 3 d_{3 / 2}$} & \multirow{2}{*}{ Te $3 d_{5 / 2}$} & \multirow{2}{*}{$\mathrm{Cd} 3 d_{3 / 2}$} & \\
\hline & $\mathrm{Te}_{\text {bulk }}$ & $\mathrm{Te}^{0}$ & $\mathrm{TeO}_{x}$ & & & & \\
\hline Исходная & 572.5 & 573.3 & 576.3 & 412.0 & 2.0 & 1.7 & 0.48 \\
\hline Обработанная в $\mathrm{HCl}-\mathrm{iPA}$ & 572.5 & 573.3 & - & 412.2 & 2.1 & 1.8 & 0.32 \\
\hline $\begin{array}{l}\text { Обработанная в } \mathrm{HCl} \text {-iPA } \\
\text { и прогретая при } 250^{\circ} \mathrm{C}\end{array}$ & 572.7 & - & - & 412.2 & 1.5 & 1.3 & 0.49 \\
\hline
\end{tabular}

энергиями 573.3 эВ и 572.5 эВ относятся к элементному теллуру $\left(\mathrm{Te}^{0}\right)$ и теллуру, связанному с $\mathrm{Cd}$ в кристаллической решетке $\left(\mathrm{Te}_{\text {bulk }}\right)$ соответственно, что хорошо согласуются с данными, полученными авторами в [24]. Линия $\mathrm{Cd} 3 d_{3 / 2}(412$ эВ) имела ширину на половине высоты 1.7 эВ и не раскладывалась на компоненты из-за малых химических сдвигов [25]. Отношение $[\mathrm{Cd}] /[\mathrm{Te}]$ на поверхности было близко к 1. На поверхности также присутствовали линии О $1 s$ и С $1 s$. По нашим оценкам, толщина оксида составляла 3-5 нм [26]. Оксидный слой на поверхности находился в аморфном состоянии.

После обработки поверхности СdTe в растворе HCl-iPA ширина линии Те $3 d_{5 / 2}$ увеличилась до 2.1 эВ и изменила форму. Линия с энергией 576.3 эВ, соответствующая оксиду, отсутствовала. Интенсивность линии с энергией 573.3 эВ, соответствующая элементному теллуру, значительно возросла относительно интенсивности линии, соответствующей $\mathrm{Te}_{\text {bulk }} \mathrm{c}$ энергией 572.5 эВ. Обогащение поверхностного слоя CdTe элементным теллуром после обработки согласуется с данными работы [27]. Энергетическое положение линии $\mathrm{Cd} 3 d_{3 / 2}$ увеличилось до 412.2 эВ с незначительным увеличением ШПВ до
1.8 эВ, что связано с удалением оксида и согласуется с данными работы [24]. Наблюдалось значительное уменьшение линии $\mathrm{C} 1 s$ и исчезновение линии О $1 s$. Таким образом, химическая обработка в $\mathrm{HCl}$-іРA приводила к существенному изменению элементного состава поверхности CdTe: удалению собственных оксидов и образованию слоя элементного теллура толщиной 3-5 нм.

Десорбция теллура с поверхности СdTе альтернативной подложки (013) GaAs/ZnTe/CdTe проводилась при термической обработке в сверхвысоком вакууме (СВВ) с контролем методами эллипсометрии и ДБЭО (рис. 2). Наблюдалось две стадии изменения состояния поверхности CdTe: низкотемпературная стадия, $\sim 125^{\circ} \mathrm{C}$, и высокотемпературная стадия, $\sim 250^{\circ} \mathrm{C}$. На первой стадии при нагреве от комнатной температуры до $125^{\circ} \mathrm{C}$ наблюдалась десорбция легколетучих адсорбированных компонентов изопропилового спирта на участке АC в плоскости изменения эллипсометрических параметров $\Psi-\Delta$. Картина дифракции поверхности (013) CdTe показала присутствие поликристаллического слоя $\mathrm{Te}^{0}$ и монокристалла CdTe. При дальнейшем нагреве происходит десорбция теллура на участке СЕ в плоско-
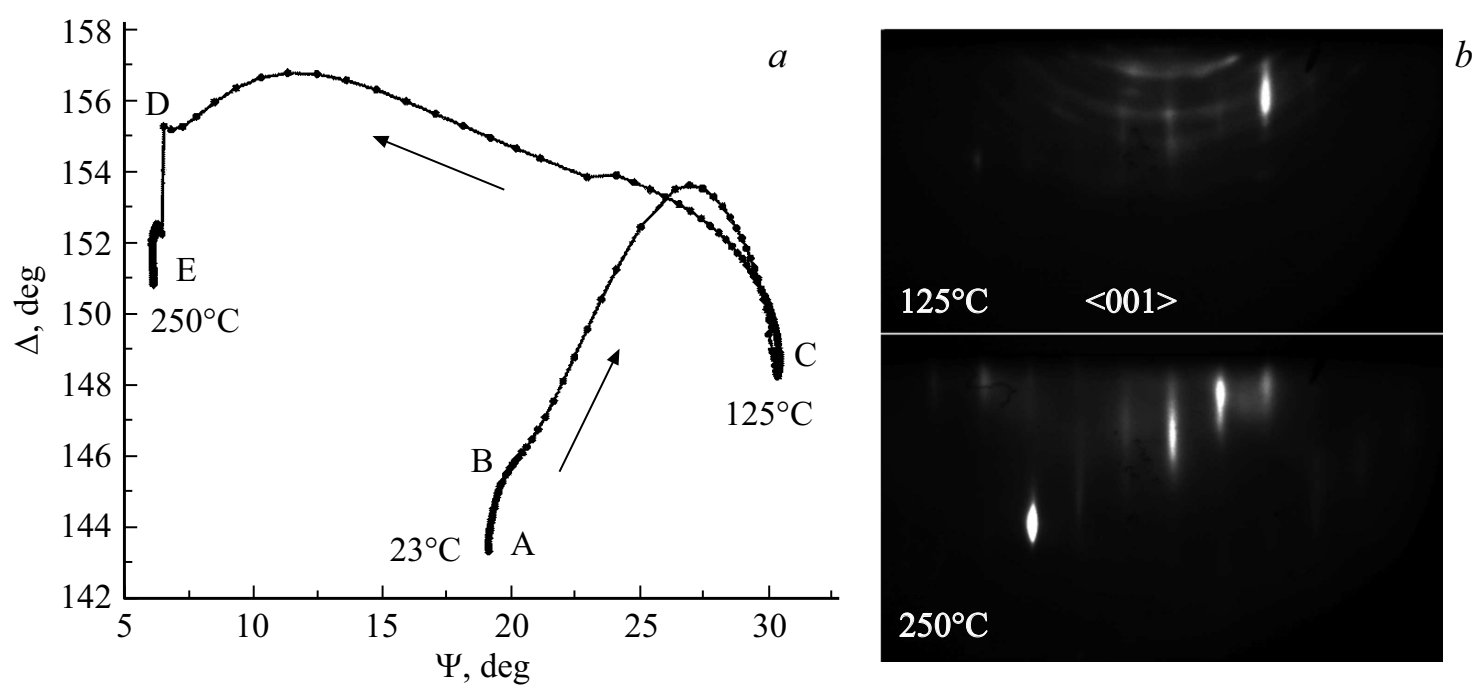

Рис. 2. Изменение состояния и структуры обработанной в HCl-iРА поверхности эпитаксиальной пленки СdTe (013) при линейном нагреве в СВВ, измеренные методами одноволновой эллипсометрии in situ $(a)$ и ДБЭО $(b)$. Стрелками показано направление изменения эллипсометрических параметров. 
$a$

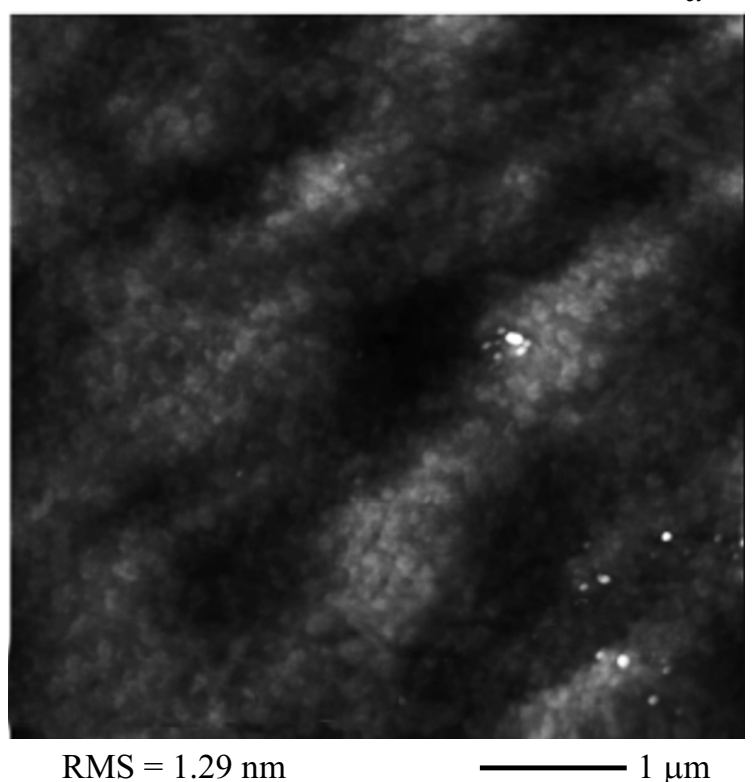

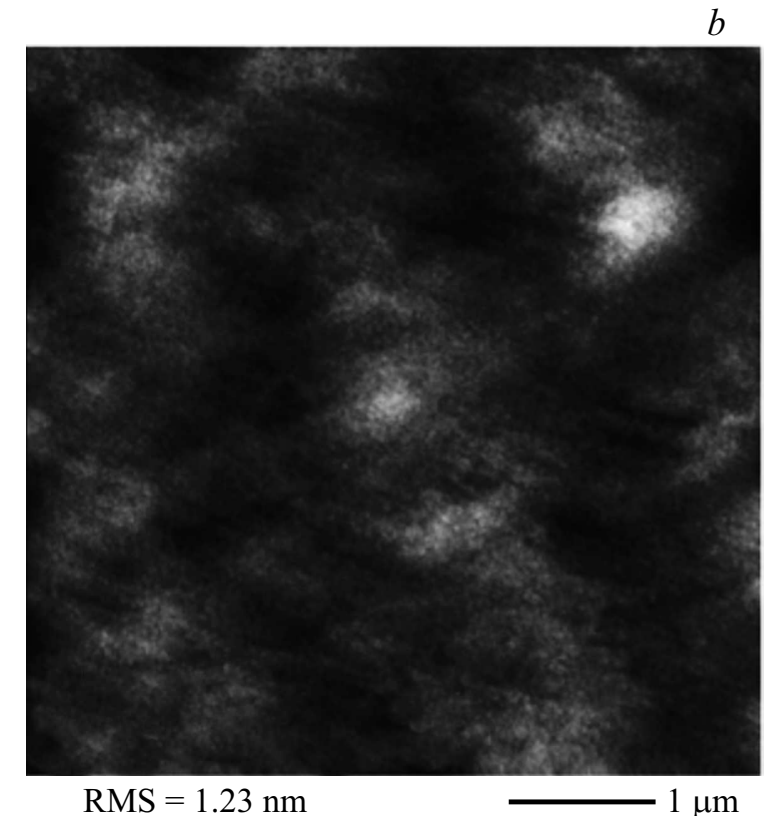

Рис. 3. АСМ-изображения поверхности эпитаксиальной пленки (013) GaAs/ZnTe/CdTe: $a-$ исходное состояние, $b-$ после обработки в $\mathrm{HCl}-\mathrm{iPA}$ и прогреве в $\mathrm{CBB}$ при температуре $250^{\circ} \mathrm{C}$. Приведены значения параметра шероховатости RMS.

сти изменения эллипсометрических параметров $\Psi-\Delta$ с появлением Те-стабилизированной поверхности (013) $\mathrm{CdTe}$, что согласуется с данными работы [28].

Значительные изменения в спектрах РФЭС наблюдались при температуре прогрева $250^{\circ} \mathrm{C}$. ШПВ линий Те $3 d_{5 / 2}$ и $\mathrm{Cd} 3 d_{3 / 2}$ уменьшились до 1.5 и 1.3 эВ соответственно. Линия, соответствующая элементному теллуру $\mathrm{Te}^{0}$, исчезла. Отношение $[\mathrm{Cd}] /[\mathrm{Te}]$ на поверхности стало близким к 1 , что свидетельствовало о получении стехиометрической поверхности. Прогрев удалял следы О, углерод регистрировался в очень малых количествах. Дальнейший прогрев не приводил к существенным изменениям в спектрах РФЭС.

Изучение поверхности методом атомно-силовой микроскопии показало, что химическая обработка и последующий прогрев практически не влияют на морфологию поверхности альтернативной подложки (013) GaAs/ZnTe/CdTe (рис. 3).

На подготовленной таким образом подложке (013) $\mathrm{GaAs} / \mathrm{ZnTe} / \mathrm{CdTe}$ (предварительно выращенный буферный слой экспонировался на воздухе более 1 года) была выращена типичная гетероэпитаксиальная структура (ГЭС) $\mathrm{Cd}_{x} \mathrm{Hg}_{1-x} \mathrm{Te}(\mathrm{KPT})$ с мольным содержанием теллурида кадмия в рабочем слое $X_{\mathrm{CdTe}}=0.194$, толщиной 5.8 мкм и варизонными слоями на гетерограницах: на гетерогранице с подложкой от $X_{\mathrm{CdTe}}=0.45$ до 0.194 на толщине 1.5 мкм, на границе с поверхностью от $X_{\mathrm{CdTe}}=0.194$ до 0.45 на толщине 0.5 мкм. При росте ГЭС КРТ не наблюдалось никаких отличительных особенностей в сравнении с ростом без экспозиции подложки на воздухе. Электрофизические параметры (температура измерения $77 \mathrm{~K}$ ) и плотность ростовых дефектов соответствовали образцам, выращенным без извлечения подложки на атмосферу и составляли: концентрация носителей заряда $n=7.6 \cdot 10^{14} \mathrm{~cm}^{-3}$, подвижность $110 \cdot 10^{3} \mathrm{~cm}^{2} / \mathrm{B} \cdot \mathrm{c}$ и время жизни неосновных носителей заряда $>1$ мкс. Плотность ростовых дефектов не превышала $500 \mathrm{~cm}^{-2}$. Электрофизические параметры измерялись методом Ван дер Пау, а время жизни неосновных носителей - по спаду СВЧ проводимости, аналогично описанному в работах $[29,30]$.

Выращенные пленки PbSnTe на подготовленных таким образом гетероструктурах (013) GaAs/ZnTe/CdTe характеризовались высоким кристаллическим совершенством и низкой шероховатостью. Рост пленок $\mathrm{PbSnTe}$ начинался сразу по послойному механизму.

\section{4. Заключение}

Получена атомарно-чистая и структурно-упорядоченная поверхность слоев СdTе в составе структуры (013) $\mathrm{GaAs} / \mathrm{ZnTe} / \mathrm{CdTe}$. Обработка поверхности CdTe в растворе HCl-iPA приводила к обогащению поверхности элементным теллуром. При температурном прогреве в СВВ наблюдалось две стадии изменения поверхности: низкотемпературная $\left(\sim 125^{\circ} \mathrm{C}\right)$ и высокотемпературная $\left(\sim 250^{\circ} \mathrm{C}\right)$, при которых происходили удаление адсорбированных компонентов, элементного теллура и формирование Те-стабилизированной поверхностности (013) CdTe.

По электрофизическим параметрам и плотности ростовых дефектов ГЭС КРТ, выращенные методом МЛЭ на подложке, экспонированной в атмосферных условиях 
в течение 1 года, с варизонными слоями на гетерограницах и толщиной рабочего слоя 5.8 мкм не уступали типичным структурам, выращиваемым в едином МЛЭпроцессе. Таким образом, показано, что подложки (013) $\mathrm{GaAs} / \mathrm{ZnTe} / \mathrm{CdTe}$ после длительного хранения в атмосфере могут быть использованы для роста высококачественных слоев CdHgTe и PbSnTe.

\section{Финансирование работы}

Выращивание ГЭС КРТ методом МЛЭ и эллипсометрические измерения проводились при финансовой поддержке гранта РФФИ № 18-29-20053. РФЭС-исследование выполнено при финансовой поддержке РФФИ и Новосибирской области в рамках научного проекта № 20-42-543015. Рост пленок $\mathrm{PbSnTe}$ проводился в рамках проекта РФФИ № 20-32-90154. АСМ-измерения проводились на оборудовании ЦКП „Наноструктуры“ при финансовой поддержке РНФ (грант № 18-72-10063).

\section{Благодарности}

Авторы выражают благодарность В.Г. Ремеснику и В.С. Варавину за проведение измерений состава и электрофизических параметров ГЭС КРТ.

\section{Конфликт интересов}

Авторы заявляют, что у них нет конфликта интересов.

\section{Список литературы}

[1] A. Rogalski. Infrared and Terahertz Detectors (Chapman and Hall/CRC, 2019).

[2] P. Liu, J.R. Williams, J.J. Cha. Nature Rev. Mater., 4, 479 (2019).

[3] C. Brüne, C.X. Liu, E.G. Novik, E.M. Hankiewicz, H. Buhmann, Y.L. Chen, X.L. Qi, Z.X. Shen, S.C. Zhang, L.W. Molenkamp. Phys. Rev. Lett., 106, 1 (2011).

[4] Е.Б. Ольшанецкий, З.Д. Квон, С.С. Кобылкин, Д.А. Козлов, Н.Н. Михайлов, С.А. Дворецкий, J.C. Portal. Письма ЖЭТФ, 93, 584 (2011).

[5] M. König, S. Wiedmann, C. Brüne, A. Roth, H. Buhmann, L.W. Molenkamp, X.L. Qi, S.C. Zhang. Science, 318, 766 (2007).

[6] З.Д. Квон, Д.А. Козлов, Е.Б. Ольшанецкий, Г.М. Гусев, Н.Н. Михайлов, С.А. Дворецкий. Успехи физ. наук, 190, $673(2020)$

[7] A.K. Kaveev, V.A. Golyashov, A.E. Klimov, E.F. Schwier, S.M. Suturin, A.S. Tarasov, O.E. Tereshchenko. Mater. Chem. Phys., 240, 122134 (2020).

[8] А.С. Тарасов, В.А. Голяшов, Д.В. Ищенко, И.О. Ахундов, А.Э. Климов, В.С. Эпов, А.К. Кавеев, В.Н. Шерстякова, С.П. Супрун, О.Е. Терещенко. Автометрия, 56, 121 (2020).

[9] В.С. Варавин, С.А. Дворецкий, Н.Н. Михайлов, В.Г. Ремесник, И.В. Сабинина, Ю.Г. Сидоров, В.А. Швец, М.В. Якушев, А.В. Латышев. Автометрия, 5, 12 (2020)

[10] H. Zogg, C. Maissen, J. Masek, T. Hoshino, S. Blunier, A.N. Tiwari. Semicond. Sci. Technol., 6, 36 (1991).
[11] В.Н. Овсюк, Г.Л. Курышев, Ю.Г. Сидоров. Матричные фотоприемные устройства инфракрасного диапазона (Новосибирск, Наука, 2001).

[12] Н.И. Филимонова, В.А. Илюшин, А.А. Величко. Автометрия, 53, 117 (2017).

[13] А.А. Величко, В.А. Илюшин, Н.И. Филимонова, Д.И. Остертак. Науч. вестн. НГТУ, 25, 131 (2006).

[14] S. Ma, C. Guo, C. Xiao, F. Wu, M. Smidman, Y. Lu, H. Yuan, H. Wu. Adv. Funct. Mater., 28 (2018).

[15] И.Г. Неизвестный, Д.В. Ищенко, И.О. Ахундов, С.П. Супрун, О.Е. Терещенко. Докл. РАН. Физика, техн. науки, 490, 39 (2020).

[16] G. Springholz, A.Y. Ueta, N. Frank, G. Bauer. Appl. Phys. Lett., 69, 2822 (1996).

[17] O.E. Tereshchenko, S.I. Chikichev, A.S. Terekhov. J. Vac. Sci. Technol. A: Vacuum, Surfaces, Films, 17, 2655 (1999).

[18] O.E. Tereshchenko, D. Paget, P. Chiaradia, J.E. Bonnet, F. Wiame, A. Taleb-lbrahimi. Appl. Phys. Lett., 82, 4280 (2003).

[19] O.E. Tereshchenko, D. Paget, P. Chiaradia, E. Placidi, J.E. Bonnet, F. Wiame, A. Taleb-Ibrahimi. Surf. Sci., 600, 3160 (2006).

[20] O.E. Tereshchenko. Appl. Surf. Sci., 252, 7684 (2006).

[21] А.Э. Климов, А.Н. Акимов, И.О. Ахундов, В.А. Голяшов, Д.В. Горшков, Д.В. Ищенко, Г.Ю. Сидоров, С.П. Супрун, А.С. Тарасов, В.С. Эпов, О.Е. Терещенко. ФТП, 53, 1207 (2019).

[22] S.A. Dvoretsky, N.N. Mikhailov, D.G. Ikusov, V.A. Kartashev, A.V. Kolesnikov, I.V. Sabinina, Yu.G. Sidorov, V.A. Shvets. In: Methods for Film Synthesis and Coating Procedures, ed. by L. Nanai, A. Samantara, S. Ratha, L. Fabian (London, InTech, 2020) p. 49.

[23] В.А. Швец, Н.Н. Михайлов, С.А. Дворецкий. Автометрия, 47, 13 (2011).

[24] A.J. Ricco, H.S. White, M.S. Wrighton. J. Vac. Sci. Technol. A: Vacuum, Surfaces, Films, 2, 910 (1984).

[25] S.S. Choi, G. Lucovsky. J. Vac. Sci. Technol. B. Microelectron. Nanom. Struct., 6, 1198 (1988).

[26] Д. Бриггс, М.П. Сих. Анализ поверхности методами Оже- и рентгеновской фотоэлектронной спектроскопии (М., Мир, 1987).

[27] S. Schreyeck, K. Brunner, L.W. Molenkamp, G. Karczewski, M. Schmitt, P. Sessi, M. Vogt, S. Wilfert, A.B. Odobesko, M. Bode. Phys. Rev. Mater., 3, 1 (2019).

[28] M.V. Yakushev, D.V. Brunev, Y.G. Sidorov. J. Surf. Investig, 4, 64 (2010).

[29] V.S. Varavin, S.A. Dvoretsky, V.I. Liberman, N.N. Mikhailov, Y.G. Sidorov. J. Cryst. Growth, 159, 1161 (1996).

[30] В.С. Варавин, С.А. Дворецкий, Н.Н. Михайлов, В.Г. Ремесник, И.В. Сабинина, Ю.Г. Сидоров, В.А. Швец, М.В. Якушев, А.В. Латышев. Автометрия, 56, 12 (2020).

Редактор Л.В. Шаронова 


\section{Preparation atomically clean and structurally ordered surfaces of epitaxial CdTe films for subsequent epitaxy}

A.S. Tarasov ${ }^{1}$, N.N. Mikhailov ${ }^{1,2}$, S.A. Dvoretsky ${ }^{1,3}$, R.V. Menshchikov ${ }^{1}$, I.N. Uzhakov ${ }^{1}$, A.S. Kozhukhov' ${ }^{1}$, E.V. Fedosenko ${ }^{1}$, O.E. Tereshchenko ${ }^{1,2}$

${ }^{1}$ Rzhanov Institute of Semiconductor Physics, Siberian Branch of Russian Academy of Sciences, 630090 Novosibirsk, Russia

${ }^{2}$ Novosibirsk State University, 630090 Novosibirsk, Russia

3 Tomsk State University, 634050 Tomsk, Russia

Abstract In this work, atomically clean and structurally ordered surface of CdTe epitaxial layer after storage in air, by treatment in isopropyl alcohol saturated with vapors of hydrochloric acid, and further temperature heating in an ultrahigh vacuum was obtained. CdTe surface chemical treatment results in the removal of native oxides and surface enrichment with elemental tellurium. Heating in vacuum led to tellurium desorption and appearance of a Te-stabilized CdTe surface. During heating in vacuum, two stages of surface state change were observed $\left(\sim 125^{\circ} \mathrm{C}\right.$ and $\leq 250^{\circ} \mathrm{C}$ ). At $T>250^{\circ} \mathrm{C}$, elemental tellurium was desorbed and a Te-stabilized structure $(1 \times 1)$ CdTe $(013)$ was formed. 